\title{
LINC computer application to research on behavioral correlates \\ of evoked neuroelectric potentials of the brain
}

W. R. GOFF and G. D. GOFF, VETERANS ADMINISTRATION HOSPITAL, West Haven, Connecticut 06516 and YALE UNIVERSITY SCHOOL OF MEDICINE, New Haven, Connecticut 06520

In this symposium so far we have heard a brief description of the history of the LINC concept, the modernization of these concepts in newer computers, and an application to clinical problems in the quantitative analysis of Parkinson's disease. I will present a use of the LINC in a more basic research application. Our laboratory is engaged in both basic and clinical research.

The purpose of the experiment that I will use to illustrate our LINC application is to examine the interaction between two kinds of cerebral evoked responses. These evoked responses are a neuroelectric reaction of the brain cells to discrete peripheral stimuli, such as a click for the auditory system or a flash for the visual system. These potentials are believed to reflect brain activity involved in stimulus detection, identification, association, and possibly even decision and memory.

The two kinds of evoked responses in which we are specifically interested are illustrated in Fig. 1. The upper schematic diagram illustrates the sensory evoked response (SER), in this case, the evoked response elicited by a transcutaneous shock stimulus to the median nerve at the wrist. Note that this schematic has a bilinear time base illustrating that the potential changes during the first 70 or $80 \mathrm{msec}$ are occurring more rapidly than the later components of the response. Also note that the total duration of this response is about $400 \mathrm{msec}$. The second type of evoked response, or perhaps it would be more accurate to use the term "event related potentials," is illustrated in the second schematic. It is the CNV, or contingent negative variation. To evoke this kind of response, an initial stimulus, $S 1$, which is a click in this particular experiment, is presented and the $S$ is told to make a

Fig. 1. Two kinds of evoked responses. The upper schematic shows the sensory evoked response (SER); the second schematic illustrates the CNV or contingent negative variation-"eventrelated potentials." response (e.g., key press) to a second stimulus, $\mathbf{S} 2$, when it occurs. $S 1$ thus serves as a "warning" or "ready" signal for a response to be made upon presentation of S2. If we are recording with nonpolarizable scalp electrodes and direct-coupled amplifiers, we will see, after the sensory evoked response to $S 1$ (seen at the beginning of the CNV trace), a steady negative dc potential, which precedes the presentation of $S 2$ and the $S$ 's response. After the response is made, the negative potential gradually returns to the baseline. Since the occurrence of the dc potential is contingent upon the presentation of S2-if you do not present $\mathbf{2} 2$, you get the sensory evoked response to $S 1$ but no dc shift-the potential has been termed the "contingent negative variation" or sometimes the "expectancy wave."

Sensory evoked responses to single stimuli are obscured by what we call the "noise" of the on-going EEG or spontaneous cortical activity. The technique of averaging neuroelectric activity following repetitive stimulus presentation to enhance the time-locked potentials of the evoked response at the expense of the randomly occurring EEG is
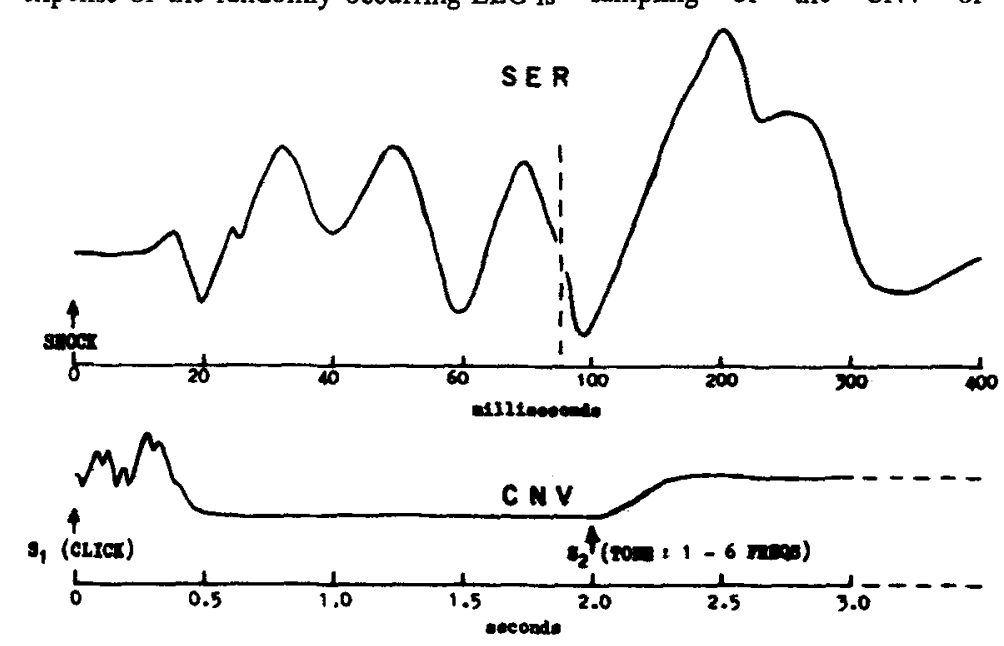

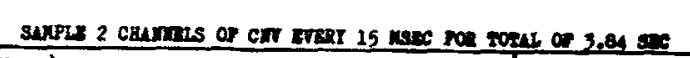
$s_{1}$ (CLICE) $s_{2}(\mathrm{rOM})$

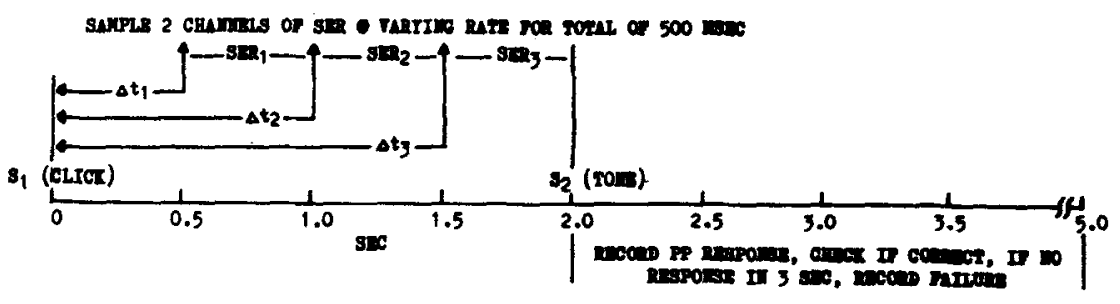

now fairly well known. Response averaging is one of our uses of the computer in the data-acquisition phase of the experiment. The CNV can be seen in the unaveraged EEG in many Ss, but it is better resolved by averaging.

In this experiment, we place nonpolarizable electrodes on the S's scalp. These are led through biological amplifiers, usually with a gain of 10,000 , directly into the LINC's analog-to-digital converter. The problem for the computer is first to summate from two electrodes, in other words, sampling and adding two A-to-D channels, starting with $S 1$, until sometime after S2. Sampling subsequent to S2 permits us to record the return of the dc potential to the base line. Since the program fills all of the lower half of the LINC's memory (1,024 registers), only the remaining half is available for data acquisition. As we need four channels of recording, two for the $\mathrm{CNV}$ and two for the SER, we can allocate only 256 memory registers, that is, one-quarter of the upper 1,024 registers of LINC memory, to each CNV channel. We use a sampling rate of every $15 \mathrm{msec}$, which gives us a total sampling of the CNV of $3.84 \mathrm{sec}$. \section{$\infty$}


Secondly, in addition to CNV sampling, we want to present a shock at three different intervals after S1 and, upon presentation of the shock, to obtain from two additional A-to-D channels an average of the sensory evoked response. It is known that the occurrence of S1 will have a suppressive effect upon the shock-evoked response as a function of the time interval between S1 and the shock. If we were to present the shock at only one fixed interval after S1, we would not know if any sensory evoked response changes were associated with the suppressive effect of $S 1$ or if they were indeed correlated with changes in the amplitude of the CNV, which is the information in which we are interested. By presenting shocks at three different fixed intervals after S1, the S1-SER suppressive effect will be varied and an analysis of variance can parcel out the effects of the suppression differentiated from effects associated with differences in the amplitude of the CNV.

For recording the SER, again we can allocate only 256 memory registers for each of the two channels. Thus, we use a varying sampling-rate program that utilizes the fact that, as I pointed out earlier, the early potentials of the evoked response are changing more rapidly than are the later ones. This varying sampling rate samples rapidly (every 500 microsec) at the beginning of the response, slows down in the middle (every $1 \mathrm{msec}$ ), and then slows down still further (every $3 \mathrm{msec}$ ) for the final components. This permits us to sample $490 \mathrm{msec}$ of activity after the shock using only 256 memory registers per channel. Thus, we can record all of the CNV and SER information needed into one-half of the LINC's memory.

Two seconds after the presentation of the S1 click stimalus, we want to present a 10-msec tone pip having one of six different frequencies. These frequencies differ by $100 \mathrm{~Hz}$, from $1,000 \mathrm{~Hz}$ to $1,500 \mathrm{~Hz}$. The tones are generated by programming the LINC's audio output and are shaped by having the LINC trigger an electronic switch that shapes the tones into the required $10-\mathrm{msec}$ tone pips without switching transients. The $S$ must identify the tone by pressing one of six keys, and this identification is sensed by the LINC over its external level lines. Performing this discrimination helps maintain the S's attention to the second stimulus, which is important both for the development of the $\mathrm{CNV}$ and because variations in attention can cause alterations in the SER. In addition, there is some evidence that the amplitude of the CNV is correlated with the accuracy of discriminative performance, and we want to gather additional information on this point.

If $3 \mathrm{sec}$ have elapsed after the presentation of $S 2$ and the $S$ has failed to respond, the computer will terminate the pass and record a response failure. The computer also determines the correctness of the S's discriminative response. It punches a paper tape that records the S1-shock interval, the tone frequency that was delivered as $\mathbf{S 2}$, the S's response, and whether or not it was correct. This punched paper tape can subsequently be entered into another program, which gives us a discriminative response profile.

It is obviously not feasible to go through the flow chart of this program in the time available. I will just enumerate the features of the program I think might be of interest. Please take into consideration that some of this involves maneuvering to get this fairly complex program and a great deal of data into the relatively small capacity of the LINC.

First of all, the program demands via the Teletype all of the relevant information needed for a response protocol, including such things as $\mathbf{S}$ identification, amount of response amplification, the number of stimulus conditions, the number of summations per stimulus condition, and so forth. Thus, when different people run the experiment, you can be sure that they get all the necessary information and that the protocol of the experiment is standardized. The program then searches the digital magnetic tape, where these evoked responses will ultimately be stored for subsequent data processing. It finds the first unused tape block and records its location. This avoids re-recording over any previously recorded data. There is an "end data tape" trap: the program computes the number of data blocks that will be needed to store all the data acquired in the experiment and, if a sufficient number are not available, it halts the computer and tells you that you need a new tape.

The program controls all the timing of stimulus presentation sequences. It samples continuously two A-to-D channels every $15 \mathrm{msec}$, from the presentation of $\mathrm{S} 1$ until approximately $2 \mathrm{sec}$ after $S 2$. It presents a shock stimulus at one of three intervals after $\mathbf{S 1}$ (there is also a fourth no-shock condition that allows us to evaluate the effect of presenting a shock per se on the CNV) and samples the sensory evoked response at the varying rate. It categorizes and stores the CNV and SER summations according to the four stimulus conditions. Two seconds after the presentation of S1, it presents $S 2$, consisting of one of six tone frequencies generated by the LINC's audio output. It senses, determines the correctness of, and records the S's discriminative responses. If there is no response in $3 \mathrm{sec}$, it records a failure. It creates a punched paper tape that allows you to evaluate subsequently the $S$ 's discriminative response profile. There is also a provision for the $\mathrm{S}$ or the $\mathrm{E}$ to interrupt the program at any time. Thus, if any problem develops in the condition of the $\mathrm{S}$ or the equipment, the program can be suspended until the problems are solved.

Because of the small memory, we cannot buffer store incoming responses; they must be added to the previous summations directly as they are sampled. Thus, between each pass, if the stimulus condition changes, all four channels of previous summations, in other words the entire upper 1,024 registers of memory, are dumped on a digital magnetic-tape working area. The previous summations for the new stimulus condition are then read into memory in preparation for adding responses to the next stimulus as they are digitalized by the A-to-D converter. Because the number of summations used in each stimulus condition would overflow the LINC's 12-bit word, summations of 16 responses (the maximum number that can be summed without overflow assuming a maximum A-to-D conversion on each pass) are temporarily stored on magnetic tape in a working area. They are subsequently recovered and entered into the average of the total number of responses, using double-register arithmetic at the end of each session. Thus, by utilizing the digital magnetic tape and punched paper tape to compensate for the small memory and small word size of the LINC, a rather complex data-acquisition program can be run and large amounts of data can be stored. Subsequent to the experiment, these data can be retrieved, quantified, analyzed, and plotted, using "piggyback" programs, some of which are four times as large as the total 2,048 registers available in the LINC's core memory. 